\title{
Principais complicações da reconstrução do trato intestinal após colostomia à Hartmann
}

\author{
Main complications of the reconstruction of the intestinal tract after colostomy to Hartmann \\ Principales complicaciones de la reconstrucción del tracto intestinal después de la \\ colostomía a Hartmann
}

\begin{abstract}
Aline Curcio de Araújo1*, Alice Magalhães Menezes², Ana Caroline Ferreira Oviedo Ramirez ${ }^{3}$, Beatriz Bandeira de Melo Kotovicz ${ }^{4}$, Camila Carolina Ueda ${ }^{5}$, Ítalo Rufino de Queiroz Fernandes ${ }^{6}$, Jéssica Reis Lopes $^{7}$, Liliana Paes Rodrigues ${ }^{8}$, Manoel Vitor Franco Dourado ${ }^{9}$, Wagner Pablo Corrêa ${ }^{10}$.
\end{abstract}

\section{RESUMO}

Objetivo: Identificar as principais complicações da reconstrução do trato intestinal após colostomia à Hartmann. Revisão bibliográfica: A Colostomia à Hartmann, é um procedimento realizado através da exteriorização do intestino grosso pela parede abdominal, para a eliminação de fezes ou gases. Esta intervenção pode ser seguida da reconstrução da continuidade do trato intestinal, através de uma anastomose colorretal para a reversão do procedimento. Por se tratar de uma técnica agressiva, sua realização pode ocasionar algumas complicações pós-operatórias, que podem inviabilizar o procedimento de reconstrução da continuidade intestinal, impactando negativamente na qualidade de vida dos pacientes. Considerações finais: Apesar de se tratar de uma técnica simples e utilizada tanto em intervenções eletivas quanto em intervenções de emergência, deve-se considerar a presença de comorbidades e a idade do paciente. Devese, ainda, considerar que a tentativa de reconstrução não deve ser realizada após muito tempo, sendo importante a adoção de medidas preventivas de educação nas etapas do pré-operatório e pós-operatório, visando a redução da ocorrência de complicações e auxiliando na melhora da qualidade de vida dos pacientes.

Palavras-chave: Colostomia, Complicações pós-operatórias, Hartmann.

\begin{abstract}
Objective: To identify the main complications of intestinal tract reconstruction after Hartmann colostomy. Bibliographic review: Hartmann's Colostomy is a procedure performed through the exteriorization of the large intestine through the abdominal wall, for the elimination of feces or gases. This intervention can be followed by the reconstruction of the continuity of the intestinal tract, through a colorectal anastomosis to reverse the procedure. Because it is an aggressive technique, its performance can cause some postoperative complications, which can make the procedure for the reconstruction of intestinal continuity unfeasible, negatively impacting the quality of life of patients. Final considerations: Although it is a simple technique and used both in elective interventions and in emergency interventions, the presence of comorbidities and the patient's age must be considered. It should also be considered that the attempt at reconstruction should not be carried out after a long time, and it is important to adopt preventive education measures in the preoperative and postoperative stages, aiming at reducing the occurrence of complications and assisting in the improvement of patients' quality of life.
\end{abstract}

Keywords: Colostomy, Postoperative complications, Hartmann.

\footnotetext{
${ }^{1}$ Instituto Tocantinense Presidente Antonio Carlos (ITPAC PORTO), Porto Nacional - TO.

*E-mail: alinecurcio@hotmail.com.br

${ }^{2}$ Faculdade de Medicina de Valença (UNIFAA), Valença - RJ.

3 Universidade de Rio Verde (UniRV), Formosa - GO.

${ }^{4}$ Centro Universitário CESMAC, Maceió - AL.

${ }^{5}$ Centro Universitário de Maringá (UNICESUMAR), Maringá - PR.

6 Universidade de Rio Verde (UniRV), Rio Verde - GO.

${ }^{7}$ Centro Universitário Tocantinense Presidente Antônio Carlos (UNITPAC), Araguaína - TO.

8 Universidade Maria Auxiliadora (UMAX), Asunción - Paraguay.

9 Universidade José do Rosário Vellano (UNIFENAS), Belo Horizonte - MG.

10 Faculdade de Medicina do Vale do Aço (UNIVAÇO), Ipatinga - MG.
} 


\section{RESUMEN}

Objetivo: Identificar las principales complicaciones de la reconstrucción del tracto intestinal tras la colostomía de Hartmann. Revisión bibliográfica: La colostomía de Hartmann es un procedimiento que se realiza mediante la exteriorización del intestino grueso a través de la pared abdominal, para la eliminación de heces o gases. Esta intervención puede ser seguida de la reconstrucción de la continuidad del tracto intestinal, mediante una anastomosis colorrectal para revertir el procedimiento. Por tratarse de una técnica agresiva, su realización puede ocasionar algunas complicaciones postoperatorias, que pueden hacer inviable el procedimiento de reconstrucción de la continuidad intestinal, impactando negativamente la calidad de vida de los pacientes. Consideraciones finales: A pesar de ser una técnica sencilla y empleada tanto en intervenciones electivas como en intervenciones de emergencia, se debe considerar la presencia de comorbilidades y la edad del paciente. También se debe considerar que el intento de reconstrucción no debe realizarse después de un tiempo prolongado, y es importante adoptar medidas de educación preventiva en la etapa preoperatoria y postoperatoria, con el objetivo de reducir la aparición de complicaciones y ayudar en la mejora de la calidad de vida de los pacientes.

Palabras clave: Colostomía, Complicaciones posoperatorias, Hartmann.

\section{INTRODUÇÃO}

A realização de uma colostomia consiste na exteriorização do intestino grosso, mais comumente do cólon transverso ou sigmoide, através da parede abdominal, para a eliminação de gases ou fezes. Nesse sentido, existe diversas técnicas que podem ser utilizadas para a realização deste procedimento. Uma dessas técnicas, é conhecida como cirurgia à Hartmann, foi realizada pela primeira vez em 1921 pelo cirurgião francês Henri Albert Hartmann. Inicialmente, o procedimento foi realizado em pacientes com câncer de cólon esquerdo obstrutivo com a finalidade de reduzir o vazamento anastomótico, ocasionado pela enfermidade anterior. Atualmente, estudos demonstram que essa técnica tende a ser agressiva, visto que as complicações podem ser inevitáveis já que possui fatores de risco como a idade ao quadro clínico apresentado pelo paciente (FONSECA AZ, et al., 2017).

O procedimento à Hartmann, com subsequente reconstrução da continuidade intestinal, é um procedimento comumente realizado. Nesse sentido, como forma de realizar a reconstrução do trato intestinal, é feita a reversão da colostomia à Hartmann por meio de uma anastomose colorretal para a promoção da saúde e do bem-estar do paciente. Contudo, a realização do procedimento é desafiador e pode apresentar reanastomose. Além disso, pesquisas afirmam taxas baixas de morbidade e mortalidade na realização tardia após 6 meses do procedimento. A intervenção cirúrgica ainda é questionável, entretanto em relação a qualidade de vida e o aumento da expectativa de vida, em conjunto com a reabilitação do paciente, resulta no seu bem-estar (MORO-VALDEZATE D, et al., 2019).

Além disso, estudos comprovam que $46 \%$ dos pacientes apresentam estoma definitivo após a cirurgia a Hartmann, fazendo com que esses pacientes não possam ser submetidos a uma segunda cirurgia. Nessa lógica, tal procedimento é considerado uma intervenção cirúrgica com taxas de morbidade de aproximadamente $58 \%$ e mortalidade de até $3,6 \%$. Os principais desafios técnicos são, geralmente, adsorção e infecção pélvica, além de dificuldades para identificar o coto retal curto. Ademais, a idade e as condições clínicas do paciente são fatores de risco para complicações no pós-operatório de cirurgia intestinais com anastomoses primárias, tanto em cirurgias de emergências como eletivas, sendo então a técnica a Hartmann uma opção para os pacientes de alto risco (HALLAM S, et al., 2018).

Acresce que a incidência de complicações na cirurgia de reconstrução do trânsito intestinal é consideravelmente elevada, uma vez que se tornou comum pacientes serem submetidos a colostomia à Hartmann principalmente em cirurgias de urgência. O paciente necessita da bolsa de colostomia, que tem a função de coletar o conteúdo fecal, o que garante um processo de evacuação de modo mais seguro. Entretanto, esse recipiente gera desconforto ao indivíduo por diminuir sua autoestima e gerar insegurança quanto a sofrer julgamentos pela sociedade. Logo, é essencial um trabalho de saúde em equipe que inclua desde o médico, que irá realizar a cirurgia, até terapeutas ocupacionais ou psicológicos, que proponham ao enfermo segurança tanto no pré quanto no pós-operatório com a perspectiva de amenizar as problemáticas 
futuras relacionadas a sua autoimagem e aos cuidados com a bolsa coletora. É importante considerar a complicação como fator preocupante já que posteriormente pode ocasionar dificuldades na qualidade de vida (AGUIAR JC, et al., 2018).

Em suma, faz-se necessário a realização de maiores estudos nessa área, a fim de minimizar os efeitos deletérios vindo das complicações do procedimento de reversão da colostomia à Hartmann. A taxa de morbidade que advém da reconstrução intestinal é considerada alta pelo excesso de doenças que se resulta após o procedimento. Estudos apontam que a produção de uma ostomia altera totalmente a qualidade de vida do enfermo. A promoção da saúde de modo integral e humanizado torna-se efetivo com a função de obter redução nas complicações e melhoria no autocuidado do doente. É fundamental um planejamento para a recuperação do paciente após o fechamento da colostomia, priorizando o bem-estar do enfermo evitando uma possível readmissão hospitalar (OLIVEIRA IV, et al.,2018).

Desta forma, o presente trabalho tem como objetivo analisar, através de uma revisão narrativa, as principais complicações da reconstrução do trato intestinal após a realização da colostomia à Hartmann.

\section{REVISÃO BIBLIOGRÁFICA}

\section{Colostomia a Hartmann}

A técnica desenvolvida por Henry Albert Hartmann, foi inicialmente descrita como uma técnica de ressecção do colón retrossigmóide, em resposta às altas taxas de complicações pós-operatórias em anastomoses colorretais em cirurgias de remoção do câncer de cólon sigmoide distal. O procedimento de Hartmann compreende em uma ressecção do retossigmóide, o fechamento do coto retal e a formação final de um estoma (HALLAM S, et al., 2018). Posteriormente, o método tornou-se procedimento de rotina utilizado como a solução de inúmeras emergências envolvendo a obstrução e perfuração do cólon (KANG JH, et al., 2020).

Apesar de haver outras alternativas de procedimentos para o controle da fonte infecciosa, como lavagem peritoneal laparoscópica ou ressecção primária com anastomose, a colostomia a Hartmann continua a ser a técnica mais utilizada na emergência atualmente, tratando obstrução do cólon por tumores malignos e diverticulite aguda com complicações (HORESH N, et al., 2020).

Comumente, encontram-se alguns desafios na cirurgia a Hartmann, incluindo o risco de retração, necrose e estenose do estoma. Tais fatores somam riscos na cirurgia de reversão, resultando na permanência do estoma em muitos pacientes. Devido a esses fatores, a colostomia a Hartmann é considerada de alto risco de mortalidade e morbidade, tornando de grande relevância a identificação de fatores preditores de riscos da cirurgia de reconstrução (HALLAM S, et al., 2018).

A realização da colostomia a Hartmann e sua reconstrução são bastante desafiadores. Uma segunda cirurgia para a reconstrução o da continuidade do intestino e o fechamento da ostomia é muito controversa, tendo em vista o alto risco de complicações pós-operatórias e as comorbidades preexistentes, encontradas frequentemente nos pacientes que são submetidos à cirurgia. Em virtude desses aspectos, muitos pacientes não podem passar por uma cirurgia de reversão, o que impacta negativamente na qualidade de vida desses (HORESH N, et al., 2020).

\section{Epidemiologia}

No Brasil existem, aproximadamente, 33.864 pessoas portadoras de ostomias; mas, esse número pode ser ainda maior, visto que essa contagem não engloba dados de todos os estados e, ainda, há muitos casos de subnotificações e ausência de cadastros nas associações estaduais (ASSOCIAÇÃO BRASILEIRA DE OSTOMIZADOS, 2017; ECCO L, et al., 2018). Dentre as colostomias, a colostomia a Hartmann é a mais comum e a mais realizada em todo o mundo (ENGIDA A, et al., 2016).

Diversos estudos, tanto no Brasil quando no mundo, mostram que mais que $50 \%$ dos pacientes que realizaram colostomia são do sexo masculino (ENGIDA A, et al., 2016; FONSECA AZ, et al., 2017; HALLAM 
S, et al., 2018). A maioria dos ostomizados no Rio Grande do Norte se autodeclarou de cor parda (47,3\%), seguidos em ordem decrescente pelos de cor branca $(38,1 \%)$, amarela $(8,2 \%)$ e negra $(5 \%)$ (ECCO L, et al., 2018). Em relação ao status econômico, estudos realizados em diferentes países - no Brasil (Rio Grande do Norte) e na África (Etiópia) - mostram que a realização da colostomia é mais frequente em indivíduos de baixa renda (ENGIDA A, et al., 2016; ECCO L, et al., 2018).

O estudo realizado no Brasil, no Rio Grande do Norte, aponta que as causas para a realização da colostomia são diversas, mas as principais são: neoplasia maligna de reto $(44,8 \%)$; neoplasia maligna de intestino (15,3\%); perfuração por arma de fogo $(5,1 \%)$; e diverticulite $(4,1 \%)$. Verificou-se, ainda, que a maioria das colostomias é temporária $(54,3 \%)$. A maioria desses pacientes com colostomia temporária possui idade até 59 anos, enquanto que entre os maiores de 59 anos a colostomia definitiva é a mais prevalente $(39,5 \%)$ (ECCO L, et al., 2018).

A média de idade dos pacientes submetidos a este procedimento cirúrgico é de 58 anos e a reconstrução do trato intestinal em pacientes com Hartmann ocorre em aproximadamente $47 \%$ dos casos, sendo que a taxa de complicação é cerca de $20,5 \%$. Estudos apontam que a reconstrução da colostomia a Hartmann é mais comum em mulheres (54\%) do que em homens (46\%) (FONSECA AZ, et al., 2017; HALLAM S, et al., 2018; WHITNEY S, et al., 2020).

A permanência hospitalar variou de 4 a 41 dias, com a média de 11,9 dias, e o tempo cirúrgico médio foi de 211,2 min. Os pacientes com complicações apresentaram tempo cirúrgico médio significantemente maior e, como esperado, os pacientes com eventos adversos (como íleo pós-operatório, vazamento anastomótico, infecção da ferida, obstrução intestinal e evisceração) apresentaram maior permanência hospitalar (FONSECA AZ, et al., 2017). As complicações mais comuns foram infecção do sítio cirúrgico (23,3\%), pneumonia adquirida no hospital (10,5\%) e deiscência da ferida (7,8\%) (ENGIDA A, et al., 2016).

A média de tempo para reconstrução da colostomia a Hartmann variou de 4 a 96 meses, tendo como média 11 meses (HALLAM S, et al., 2018). Estudos apontam, ainda, que a taxa de morbidade associada à reconstrução do estoma é de aproximadamente $50 \%$ e o risco de mortalidade é maior que $5 \%$ (ROIG JV, et al., 2016). Porém, outro estudo observou taxa de mortalidade mais elevada $(9,5 \%)$ devido à apresentação tardia e comorbidades associadas; por isso, na maioria das vezes, este procedimento é realizado em condições de emergência (ENGIDA A, et al., 2016).

\section{Reversões}

Ademais, em relação ao período ideal para a realização da reversão do procedimento de colostomia a Hartmann, ainda não há certeza do momento mais indicado para tal. Contudo, estudos que abordam sobre reconstruções precoces ou tardias mostraram que o período entre os procedimentos variou entre intervalos de 4,6 e 8 meses. Nesse sentido, tem-se o argumento de apoio à reversão a Hartmann tardio sustentado com base na necessidade de permitir a diminuição de edema, inflamação e sepse de modo que a melhora do estado clínico e nutricional do paciente resulte em menos complicações. Por outro lado, alguns estudos relatam que há aumento das complicações quando se tem um período maior de intervalo entre a colostomia e a reversão. Nesse sentido, eles relacionam esse entremeio à atrofia do coto distal e, em vista disso, aumentam a dificuldade de realização da anastomose (HALLAM S, et al., 2018).

Diante disso, alguns fatores são predominantes na decisão do momento certo para a realização desse procedimento. Os pacientes que são submetidos à cirurgia a Hartmann, em função de sepse intra-abdominal, estão aptos a reversão somente quando a sepse é inteiramente tratada, levando em consideração os fatores que ocasionaram a infecção. Em pacientes que são sujeitos a colostomia devido à obstrução maligna do cólon, deve-se considerar que o tratamento está associado à abordagem oncológica adjuvante, a qual impacta expressivamente no fechamento do estoma e, consequentemente, no tempo para a realização da reversão (HORESH N, et al., 2020).

O tipo de procedimento realizado na cirurgia de reversão está intimamente ligado ao intervalo entre as cirurgias e às complicações pós-operatórias. Diante disso, na cirurgia aberta, o intervalo de tempo entre o procedimento inicial e a reversão é de cerca de 6 meses, uma vez que estudos mostram que taxas de 
complicações pós-operatórias tendem a aumentar à medida que se atrasa o processo de reversão. Por outro lado, com a abordagem laparoscópica, o intervalo de tempo entre os procedimentos é comumente mais curto. A liberação cirúrgica de aderências abdominais consequentes de cirurgias anteriores pode ser tecnicamente desafiadora e a cirurgia é mais fácil quando menos aderências são encontradas, justificando assim um período de espera mais curto, uma vez que quanto mais tempo esperar para a reversão mais aderências tendem a se formar (LUCCHETTA A e DE MANZINI N, 2016).

Visando o sucesso no procedimento cirúrgico, é indispensável um adequado preparo do coto retal, sendo que a dificuldade de visualizar corretamente o coto é uma causa importante de conversão malsucedida. Tal problema está relacionado com a atrofia retal que ocorre, em média, após 8 a 10 semanas do procedimento inicial. Dessa maneira, visando minimizar tal problema, alguns autores sugerem deixar uma sutura de polipropileno no reto para facilitar na sua identificação ou usar dilatadores retais durante a dissecção do coto (LUCCHETTA A e DE MANZINI N, 2016).

Não há consenso na literatura sobre a abordagem mais indicada, contudo muitos autores destacaram alguns benefícios da abordagem laparoscópica em comparação à técnica aberta, entre eles: menor perda de sangue intra-operatória, menor tempo de internação, redução da dor pós-operatória, tempo reduzido para a primeira evacuação, menos complicações pós-operatórias e menor taxa de hérnias incisionais (LUCCHETTA A e DE MANZINI N, 2016).

Por outro lado, um estudo retrospectivo realizado no Hospital de Bremerhaven, na Alemanha, revelou que a técnica VULKAN para fechamento de colostomia tem mostrado resultados positivos em relação às taxas de infecções no local cirúrgico e produz bons resultados cosméticos. Nesta técnica, a reanastomose intestinal é seguida pelo fechamento circular do tecido subcutâneo em camadas com a retenção de um pequeno defeito secundário através do qual os fluidos exsudativos e supurativos podem ser drenados. Dessa forma, faz-se o fechamento externo da mucosa intestinal para evitar derramamento fecal seguido pela incisão circular em torno da ostomia para liberar as aderências e mobilizar o intestino, realiza-se a anastomose costurada ou grampeada manualmente e, por fim, tem-se o fechamento da parede suturada com polidioxanona em espessura total (KRENZIEN F, et al., 2017).

\section{Complicações}

Um primeiro estudo que avaliou 39 pacientes que realizaram o procedimento a Hartmann observou que, destes, oito tiveram complicações consideráveis, sendo que o efeito adverso mais comum encontrado foi a deiscência anastomótica, (37,5\%), seguido de deiscência e evisceração (10\%), obstrução intestinal (10\%), infecção da ferida operatória (10\%) e impossibilidade de reconstrução (10\%). Dos oito pacientes que apresentaram problemas na cirurgia, três evoluíram a óbito, sendo que dois deles vieram a óbito por complicações de origem infecciosa e disfunção multiorgânica devido a uma lesão no intestino delgado, e um por complicações pulmonares (FONSECA AZ, et al., 2017).

Em outro estudo, verificou-se que, dos 127 indivíduos que realizaram procedimento de reversão do procedimento a Hartmann, 46 pacientes $(35,9 \%)$ apresentaram complicações importantes, como íleo paralítico e infecção da ferida, sendo que $8(6,3 \%)$ deles apresentaram mais de uma. A complicação mais comum foi o íleo paralítico (13,3\%), que é causado principalmente por aderências graves na cavidade abdominal, seguida de infecção da ferida $(9,4 \%)$, dados semelhantes à do estudo de Fonseca $A Z$, et al. (2017). Por fim, outra complicação verificada foi hérnia incisional no local da colostomia $(8,6 \%)$. Em outra análise, a complicação mais comum após cirurgia a Hartmann foi a infecção da ferida, implicando em cerca de $16,6-40 \%$ dos casos (KANG JH, et al., 2020).

Outro estudo analisado, observou-se que de 228 pacientes que realizaram cirurgia a Hartmann, 23 tiveram complicações. A infecção da ferida foi a complicação mais comum, sendo considerada em $8 \%$ dos casos. Além disso, complicações de Clavien-Dindo de grau III ou acima desse, ocorreram em cerca de $5 \%$ dos pacientes, dos quais todos eram de deiscência anastomótica. Fatores associados às complicações após a reversão incluem o tabagismo, visto que $52 \%$ dos fumantes apresentaram complicações, e tempo de internação superior a 11 dias, pois $40 \%$ desses pacientes apresentaram problemas significativos após a cirurgia (HALLAM S, et al., 2018). 
O momento do fechamento dos estômatos ainda é controverso. Existem duas tendências principais: aqueles que acreditam que a reconstrução precoce será melhor, e aqueles que desejam realizar a cirurgia em seus pacientes após um longo período de tempo após a cirurgia. O último grupo acredita que o melhor momento é depois que a inflamação abdominal do paciente é completamente aliviada e uma nutrição melhor é obtida. Um curto tempo de reversão pode prevenir a atrofia do coto retal. No entanto, alguns grupos não encontraram diferença nas taxas de reversão e taxas de complicações entre esses dois períodos (FONSECA AZ, et al., 2017).

Com os estudos analisados, foi possível perceber que a maioria dos pacientes que tiveram complicações, apresentaram principalmente infecção de ferida, deiscência anastomótica e evisceração após a colostomia a Hartmann e isso ocorre, principalmente, devido ao fato de que esses pacientes apresentavam idade avançada e comorbidades pré-existentes, como neoplasias do cólon e diverticulite, diferente daqueles que por serem jovens e não terem doenças precedentes, não tiveram complicações (FONSECA AZ, et al., 2017).

A idade pode ser considerada como fator de risco, visto que, normalmente, pacientes mais velhos necessitam de estomas devido ao câncer, estado esse que gera o agravamento da saúde e, por consequência, mais complicações. Já os pacientes jovens, tendem a ser mais saudáveis além de terem um índice de recuperação maior em relação aos idosos. Dessa forma, as pessoas que foram submetidas a colostomia a Hartmann e que não reconstruíram o trânsito devido à comorbidades apresentaram idade superior quando comparadas com as demais causas. Fisiologicamente o envelhecimento do indivíduo vem acompanhado pelo desenvolvimento de condições crônicas de saúde e essas morbidades podem aumentar significativamente a morbimortalidade na reconstrução do trânsito, culminando na não realização do procedimento em virtude de apresentarem maiores riscos (AGUIAR JC, et al., 2018; FONSECA AZ, et al., 2017).

Por fim, os pacientes que necessitaram de transfusão sanguínea, internação em UTI e que tiveram um tempo de operação mais longo que outros enfermos, foram associados a uma maior taxa de complicações. Como a cirurgia a Hartmann é um procedimento de opção dos cirurgiões frente a pacientes mais debilitados, a sua utilização em pacientes com abdômen agudo perfurativo, obstrutivo ou inflamatório, depende muito da experiência de cada cirurgião na indicação da sua realização (FONSECA AZ, et al., 2017; KANG JH, et al., 2020).

Um dos fatores que poderia reduzir a incidência de complicações é a reversão laparoscópica. Este método pode diminuir a prevalência de complicações, reduzir a perda de sangue, além de encurtar o tempo de internação hospitalar e acelerar o retorno dos movimentos intestinais (FONSECA AZ, et al., 2017).

\section{CONSIDERAÇÕES FINAIS}

Por conseguinte, a cirurgia à Hartmann é muito utilizada tanto em cirurgias realizadas na urgência, quanto de forma eletiva. Os aspectos relacionados a qualidade de vida devem ser considerados, já que o paciente requer o uso de uma bolsa de colostomia, gerando constrangimento e desconforto ao indivíduo e levando a diminuição da autoestima pela reprovação física dentro da sociedade. Além disso, é importante criar medidas de acompanhamento antes e após o ato cirúrgico, pois existem dificuldades do paciente e da família no manejo da colostomia. Medidas preventivas de educação em saúde no pré-operatório e no pós-operatório ajudariam evitar complicações, melhorando assim a qualidade de vida dos pacientes após o procedimento, diminuindo a ansiedade da família e reduzindo custos orçamentários para hospitais.

\section{REFERÊNCIAS}

1. AGUIAR JC, et al. Reconstrução de trânsito intestinal: fatores que influenciam a realização. Revista Eletrônica de Enfermagem, 2018.

2. ANTOLOVIC D, et al. Restoration of intestinal continuity after Hartmann's procedure - not a benign operation. Are there predictors for morbidity? Langenbeck's Archives of Surgery, 2011; 396 (7): 989-996.

3. AQUINA CT, et al. The impact of surgeon volume on colostomy reversal outcomes after Hartmann's procedure for diverticulitis. Surgery, 2016; 160 (5): 1309-1317. 
4. ASSOCIAÇÃO BRASILEIRA DE OSTOMIZADOS. Quantitativo aproximado de pessoas ostomizadas no Brasil, 2017.

5. ASSIS F, CANUTO HA. Perfil Dos Pacientes Submetidos À Reconstrução De Trânsito Intestinal No Ano De 2015 No Hospital Geral De Fortaleza, 2017.

6. BRATHWAITE S, et al. Risk Factors for Surgical Site Infection in Open and Laparoscopic Hartmann Closure. Surgical Laparoscopy, Endoscopy \& Percutaneous Techniques, 2017; 27 (1): 51-53.

7. CAMPOS K, et al. The impact of colostomy on the patient's life. Journal of Coloproctology, $2017 ; 37$ (3): $205-210$.

8. CHRISTOU N, et al. Identification of risk factors for morbidity and mortality after Hartmann's reversal surgery - a retrospective study from two French centers. Scientific Reports, 2020; 10 (1).

9. ECCO L, et al. Perfil de pacientes colostomizados na Associação dos Ostomizados do Rio Grande do Norte. ESTIMA, Brazilian Journal of Enterostomal Therapy, 2017.

10. ENGIDA A, et al. Types and Indications of Colostomy and Determinants of Outcomes of Patients After Surgery. Ethiopian Journal of Health Sciences, 2016; 26 (2): 117.

11. FONSECA AZ, et al. Colostomy Closure: Risk Factors For Complications. Arquivos Brasileiros de Cirurgia Digestiva, 2017; 30 (4): 231-234.

12. NASCIMENTO MVF, et al. Perfil Sociodemográfico E Clínico De Pacientes Em Pós-Operatório De Confecção De Estomas Intestinais De Eliminação. Ciencia y enfermería, 2018.

13. HALLAM S, et al. Hartmann's procedure, reversal and rate of stoma-free survival. The Annals of The Royal College of Surgeons of England, 2018; 100 (4): 301-307.

14. HORESH N, et al. Reversal of Hartmann's procedure: still a complicated operation. Techniques in Coloproctology, 2017; 22 (2): 81-87.

15. HORESH N, et al. Timing of colostomy reversal following Hartmann's procedure for perforated diverticulitis. Journal of Visceral Surgery, 2020; 157 (5): 395-400.

16. KANG JH, et al. Analysis of factors affecting reversal of Hartmann's procedure and post-reversal complications. Scientific Reports, 2020; 10 (1).

17. KRENZIEN F, et al. The Vulkan Technique: A Novel Ostomy-Closure Technique That Reduces Complications And Operative Times. ABCD - Arquivos Brasileiros de Cirurgia Digestiva, 2017; 30 (2): 139-142.

18. LUCCHETTA A, DE MANZINI N. Laparoscopic reversal of Hartmann procedure: is it safe and feasible? Updates in Surgery, 2016; 68 (1): 105-110.

19. MORO-VALDEZATE D, et al. Outcomes of Hartmann's procedure and subsequent intestinal restoration. Which patients are most likely to undergo reversal? The American Journal of Surgery, 2019; 218 (5): 918-927.

20. ONDER A, et al. Comparison of Short-term Outcomes After Laparoscopic Versus Open Hartmann Reversal. Surgical Laparoscopy, Endoscopy \& Percutaneous Techniques, 2016; 26 (4): 75-79.

21. ROIG JV, et al. Restoration of Bowel Continuity After Emergency Hartmann's Procedure. Journal of Colon And Rectal Cancer, 2016; 1 (2): 7-16.

22. SCIUTO P, et al. Restitución del procedimiento de Hartmann por vía laparoscópica. Análisis de nuestra experiencia en el Hospital Maciel de Montevideo, Uruguay, Clínicas Quirúrgicas 2 y 3 de la Facultad de Medicina de la Universidad de la República. Revista Chilena de Cirugía, 2017; 69 (6): 446-451.

23. STABILINI C, GIANETTA E. Parastomal Hernia Prevention and Treatment. The Art of Hernia Surgery, 2018.

24. TEI M, et al. Evaluation of postoperative pain at the stoma site in patients who underwent a single-port Hartmann's procedure. Asian Journal of Endoscopic Surgery, 2015; 8 (4): 424-428.

25. TSUCHIYA A, et al. Mortality and Morbidity After Hartmann's Procedure Versus Primary Anastomosis Without a Diverting Stoma for Colorectal Perforation: A Nationwide Observational Study. World Journal of Surgery, $2017 ; 42$ (3): 866-875.

26. OLIVEIRA IV, et al. Cuidado e saúde em pacientes estomizados. Revista Brasileira em Promoção da Saúde, $2018 ; 31$ (2): $1-9$.

27. WHITNEY S, et al. Hartmann's reversal: factors affecting complications and outcomes. International Journal of Colorectal Disease, 2020; 35 (10) 1875-1880. 